\title{
FUNCTIONS WITH THE HUYGENS PROPERTY
}

\section{BY DEBORAH TEPPER HAIMO ${ }^{1}$}

Communicated by A. Zygmund, January 11, 1965

A $C^{2}$ function $u(x, t)$ belongs to class $H$, for $a<t<b$, and is called a generalized temperature function, if and only if it is a solution of the generalized heat equation

$$
\Delta_{x} u(x, t)=(\partial / \partial t) u(x, t),
$$

where $\Delta_{x} f(x)=f^{\prime \prime}(x)+(2 \nu / x) f^{\prime}(x), \nu$ a fixed positive number. The fundamental solution of this equation is

$$
G(x, y ; t)=(1 / 2 t)^{p+1 / 2} g(x y / 2 t) \exp \left[-\left(x^{2}+y^{2}\right) / 4 t\right],
$$

with $\mathscr{I}(z)=c_{\nu} z^{1 / 2-\nu} I_{\nu-1 / 2}(z), c_{\nu}=2^{\nu-1 / 2} \Gamma\left(\nu+\frac{1}{2}\right)$, and $I_{\gamma}(z)$ the Bessel function of order $\gamma$ of imaginary argument. We write $G(x ; t)$ for $G(x, 0 ; t)$. The function $u(x, t)$ is said to have the Huygens property, that is, it belongs to class $H^{*}$, for $a<t<b$, if and only if $u(x, t) \in H$ there, and

$$
u(x, t)=\int_{0}^{\infty} G\left(x, y ; t-t^{\prime}\right) u\left(y, t^{\prime}\right) d \mu(y), \quad d \mu(x)=\left(1 / c_{v}\right) x^{2 v} d x,
$$

for every $t, t^{\prime}, a<t^{\prime}<t<b$, the integral converging absolutely. A generalized heat polynomial $P_{n, \nu}(x, t)$ is defined by

$$
P_{n, \nu}(x, t)=\sum_{k=0}^{n} 2^{2 k}\left(\begin{array}{l}
n \\
k
\end{array}\right)\left[\Gamma\left(\nu+\frac{1}{2}+n\right) / \Gamma\left(\nu+\frac{1}{2}+n-k\right)\right] x^{2 n-2 k} t^{k},
$$

and its Appell transform $W_{n, \nu}(x, t)$ is given by

$$
W_{n, v}(x, t)=G(x, t) P_{n, v}(x / t,-1 / t) .
$$

The object of this paper is to summarize the principal results derived in characterizing a generalized temperature function which may be represented either by the series expansion $\sum_{n=0}^{\infty} a_{n} P_{n, \nu}(x, t)$ or by $\sum_{n=0}^{\infty} b_{n} W_{n, v}(x, t)$, with convergence taken in the $L^{2}$, as well as in the pointwise, sense. Details and proofs will appear later. The work is an extension of the theory developed by Rosenbloom and Widder in [3]. Some of the preliminary results for this study were also de-

1 The research was supported by a National Science Foundation fellowship at Harvard University. 
rived by F. M. Cholewinski, and it has been called to our attention that Louis Bragg has done work in this area.

The region of convergence of the series $\sum_{n=0}^{\infty} a_{n} P_{n, v}(x, t)$ is, in general, a strip $|t|<\sigma$, whereas that of the series $\sum_{n=0}^{\infty} b_{n} W_{n, v}(x, t)$ is a half plane $t>\sigma \geqq 0$. Indeed, we have

Theorem 1. If $\lim _{n \rightarrow \infty}\left|a_{n}\right|^{1 / n} 4 n / e=1 / \sigma<\infty$, then the series

$$
\sum_{n=0}^{\infty} a_{n} P_{n, \nu}(x, t)
$$

converges absolutely in the strip $|t|<\sigma$ and does not converge everywhere in any including strip.

TheOREM 2. If $\lim _{n \rightarrow \infty}\left|b_{n}\right|^{1 / n} 4 n / e=\sigma<\infty$, then the series

$$
\sum_{n=0}^{\infty} b_{n} W_{n, \nu}(x, t)
$$

converges absolutely in the half plane $t>\sigma \geqq 0$ and does not converge everywhere in any including half plane.

Within their regions of convergence, $\sum_{n=0}^{\infty} a_{n} P_{n, \nu}(x, t)$ and $\sum_{n=0}^{\infty} b_{n} W_{n, \nu}(x, t)$ each defines a generalized temperature function $u(x, t)$, with the additional fact, in the first case, that $u(x, 0)$ is an even entire function of growth $(1,1 / 4 \sigma)$. An entire function $\phi(x)$ $=\sum_{n=0}^{\infty} c_{n} x^{n}$ is said to be of growth $(\rho, \tau)$ if and only if $\lim _{n \rightarrow \infty} n\left|c_{n}\right| \rho / n$ $\leqq \tau e \rho$.

Since we find that $u(x, t)$ has an expansion $\sum_{n=0}^{\infty} a_{n} P_{n, v}(x, t)$ in the largest strip $|t|<\sigma$ for which $u(x, t) \in H^{*}$, we note that the role of membership in class $H^{*}$ in expansions in terms of generalized heat polynomials is analogous to that of analyticity in expansions in Taylor series. This is established in the following.

Theorem 3. A necessary and sufficient condition that

$$
u(x, t)=\sum_{n=0}^{\infty} a_{n} P_{n, v}(x, t),
$$

the series converging for $|t|<\sigma$, is that $u(x, t) \in H^{*}$ there. The coeffcients $a_{n}$ have either of the determinations

$$
\begin{gathered}
a_{n}=u^{(2 n)}(0,0) /(2 n) !, \text { or } \\
a_{n}=\left\{\Gamma\left(\nu+\frac{1}{2}\right) /\left[2^{4 n} n ! \Gamma\left(\nu+\frac{1}{2}+n\right)\right]\right\} \int_{0}^{\infty} u(y,-t) W_{n, \nu}(y, t) d \mu(y), \\
0<t<\sigma .
\end{gathered}
$$


In addition, the following result gives us a complex determination of the coefficients.

THEOREM 4. If $u(x, t)=\sum_{n=0}^{\infty} a_{n} P_{n, v}(x, t)$, the series converging for $|t|<\sigma$, then

$$
\begin{array}{r}
a_{n}=\left\{(-1)^{n} \Gamma\left(\nu+\frac{1}{2}\right) /\left[2^{4 n} n ! \Gamma\left(\nu+\frac{1}{2}+n\right)\right]\right\} \int_{0}^{\infty} u(i x, t) W_{n, \nu}(x, t) d \mu(x), \\
0<t<\sigma .
\end{array}
$$

Membership in class $H^{*}$ is not sufficient for an expansion in terms of $W_{n, \nu}(x, t)$, as is indicated by the function $u(x, t)=1$, which is in $H^{*}$ for $-\infty<x<\infty$ but cannot be represented by the expansion $\sum_{n=0}^{\infty} b_{n} W_{n, \nu}(x, t)$. Instead we have the following modification of the dual to Theorem 3.

Theorem 5. A necessary and sufficient condition that

$$
u(x, t)=\sum_{n=0}^{\infty} b_{n} W_{n, v}(x, t),
$$

the series converging for $t>\sigma \geqq 0$, is that $u(x, t) \in H^{*}$ there and that

$$
\int_{0}^{\infty}|u(x, t)| e^{x^{2} / 8 t} d \mu(x)<\infty, \quad \sigma<t<\infty .
$$

The coefficients $b_{n}$ have the determination

$$
\begin{array}{r}
b_{n}=\left\{\Gamma\left(\nu+\frac{1}{2}\right) /\left[2^{4 n} n ! \Gamma\left(\nu+\frac{1}{2}+n\right)\right]\right\} \int_{0}^{\infty} u(y, t) P_{n, \nu}(y,-t) d \mu(y), \\
\sigma<t<\infty .
\end{array}
$$

The proof of this theorem depends on one which establishes necessary and sufficient conditions of a different nature for such an expansion.

TheOREm 6. A necessary and sufficient condition that

$$
u(x, t)=\sum_{n=0}^{\infty} b_{n} W_{n, \nu}(x, t),
$$

the series converging for $t>\sigma \geqq 0$, is that

$$
u(x, t)=\int_{0}^{\infty} \mathcal{J}(x u) e^{-t y^{2}} \phi(y) d \mu(y), \quad \sigma<t<\infty,
$$

where $\mathcal{J}(z)=2^{\nu-1 / 2} \Gamma(\nu+1 / 2) z^{1 / 2-\nu} J_{\nu-1 / 2}(z), \phi(y)$ is an even entire function of growth $(1, \sigma)$, and $b_{n}=\phi^{(2 n)}(0) /\left[(2 n) !(-4)^{n}\right]$. 
For expansions in the $L^{2}$ sense, we have the following:

Theorem 7. If $u(x, t) \in H^{*},-\sigma \leqq t<0$, and if $u(x ; t)[G(x ;-t)]^{1 / 2}$ $\in L^{2}$, for each fixed $t,-\sigma \leqq t<0,0 \leqq x<\infty$, then, for $-\sigma \leqq t<0$,

$$
\lim _{N \rightarrow \infty} \int_{0}^{\infty} G(x,-t)\left|u(x, t)-\sum_{n=0}^{N} a_{n} P_{n, \nu}(x, t)\right|^{2} d \mu(x)=0,
$$

and

$$
\int_{0}^{\infty} G(x,-t)|u(x, t)|^{2} d \mu(x)=\sum_{n=0}^{\infty} 2^{4 n} n ! \Gamma\left(\nu+\frac{1}{2}+n\right)\left|a_{n}\right|{ }^{2} t^{2 n} / \Gamma\left(\nu+\frac{1}{2}\right),
$$

where

$$
\begin{array}{r}
a_{n}=\left\{\Gamma\left(\nu+\frac{1}{2}\right) /\left[2^{4 n} n ! \Gamma\left(\nu+\frac{1}{2}+n\right)\right]\right\} \int_{0}^{\infty} u(y, t) W_{n, \nu}(y,-t) d \mu(y), \\
-\sigma \leqq t<0 .
\end{array}
$$

The example $u(x, t)=e^{a^{2} \operatorname{tg}(a x)}$ illustrates a limitation of this theorem. Although, in this case, $u(x, t) \in H^{*}$ for $0<t<\infty$, as well as for $-\infty<t \leqq 0$, so that it may be represented by $\sum_{n=0}^{\infty} a_{n} P_{n, v}(x, t)$, with convergence in the pointwise sense for $0<t<\infty$, Theorem 7 fails to give such an expansion in the $L^{2}$ sense for $0<t<\infty$, since $u(x, t)[G(x,-t)]^{1 / 2} \in L^{2}$ for $0<t<\infty$. Thus, for $t>0$, we need an additional result.

Theorem 8. If $u(x, t) \in H^{*}, 0<t \leqq \sigma$, and if $u(i x, t)[G(x, t)]^{1 / 2} \in L^{2}$, for each fixed $t, 0<t \leqq \sigma, 0 \leqq x<\infty$, then, for $0<t \leqq \sigma$,

$$
\lim _{N \rightarrow \infty} \int_{0}^{\infty} G(x, t)\left|u(i x, t)-\sum_{n=0}^{N} a_{n} P_{n, \nu}(x,-t)\right|^{2} d \mu(x)=0,
$$

and

$$
\int_{0}^{\infty} G(x, t)|u(i x, t)|^{2} d \mu(x)=\sum_{n=0}^{\infty} 2^{4 n} n ! \Gamma\left(\nu+\frac{1}{2}+n\right)\left|a_{n}\right|^{2} t^{2 n} / \Gamma\left(\nu+\frac{1}{2}\right),
$$

where

$$
\begin{array}{r}
a_{n}=\left\{\Gamma\left(\nu+\frac{1}{2}\right) /\left[2^{4 n} n ! \Gamma\left(\nu+\frac{1}{2}+n\right)\right]\right\} \int_{0}^{\infty} u(i x, t) W_{n, \nu}(x, t) d \mu(x), \\
0<t \leqq \sigma .
\end{array}
$$

The dual to Theorem 7 is the following: 
Theorem 9. If $u(x, t) \in H^{*}, t \geqq \sigma>0$, and if $u(x, t)[G(i x, t)]^{1 / 2} \in L^{2}$, for each fixed $t, t \geqq \sigma>0,0 \leqq x<\infty$, then for $t \geqq \sigma>0$,

$$
\lim _{N \rightarrow \infty} \int_{0}^{\infty} G(i x, t)\left|u(x, t)-\sum_{n=0}^{N} b_{n} W_{n, \nu}(x, t)\right|^{2} d \mu(x)=0,
$$

and

$$
\begin{aligned}
\int_{0}^{\infty} G(i x, t) \mid u(x, t) & \left.\right|^{2} d \mu(x) \\
& =\sum_{n=0}^{\infty} 2^{4 n-2 \nu-1} n ! \Gamma\left(\nu+\frac{1}{2}+n\right)\left|a_{n}\right|^{2 t-2 n-2 \nu-1} / \Gamma\left(\nu+\frac{1}{2}\right),
\end{aligned}
$$

where

$$
\begin{array}{r}
b_{n}=\left\{\Gamma\left(\nu+\frac{1}{2}\right) /\left[2^{4 n} n ! \Gamma\left(\nu+\frac{1}{2}+n\right)\right]\right\} \int_{0}^{\infty} u(x, t) P_{n, \nu}(x,-t) d \mu(x), \\
0 \leqq t<\infty .
\end{array}
$$

The properties of $P_{n, v}(x, t)$ and $W_{n, \nu}(x, t)$ play a central role in the development of the theory. Of primary importance is the fact that the polynomials $P_{n, \nu}(x, t)$ and the functions $W_{n, \nu}(x, t)$ form a biorthogonal system in the sense that

$$
\int_{0}^{\infty} W_{n, \nu}(x, t) P_{m, \nu}(x,-t) d \mu(x)=\delta_{m n} 2^{4 n} n ! \Gamma\left(\nu+\frac{1}{2}+n\right) / \Gamma\left(\nu+\frac{1}{2}\right) .
$$

In addition, the equation

$$
G(x, y ; s-t)=\sum_{n=0}^{\infty}\left\{\Gamma\left(\nu+\frac{1}{2}\right) /\left[2^{4 n} n ! \Gamma\left(\nu+\frac{1}{2}+n\right)\right]\right\} W_{n, \nu}(y, s) P_{n, \nu}(x,-t)
$$

is fundamental. We make repeated use, in the proofs, of asymptotic estimates of $P_{n, \nu}(x, t)$ and $W_{n, \nu}(x, t)$. Indeed, these estimates, in addition to the fact that $P_{n, \nu}(x, t) \in H^{*}$, for $-\infty<t<\infty$, and $W_{n, \nu}(x, t)$ $\in H^{*}$, for $0<t<\infty$, enable us to prove that the integral determinations of the coefficients in the above series expansions are all independent of $t$.

\section{REFERENCES}

1. F. M. Cholewinski and D. T. Haimo, The Weierstrass-Hankel convolution transform, J. Analyse Math. (to appear).

2. D. T. Haimo, Generalized temperature functions, Duke Math. J. (to appear).

3. P. C. Rosenbloom and D. V. Widder, Expansions in terms of heat polynomials and associated functions, Trans. Amer. Math. Soc. 92 (1959), 220-266.

HARVARD UNIVERSITY 